\title{
Trabalho voluntário em saúde: auto-percepção, estresse e burnout
}

\author{
André Luís Ferreira Moniz. \\ Faculdade Cenecista de Brasília \\ Tereza Cristina Cavalcanti Ferreira de Araujo \\ Universidade de Brasília
}

\begin{abstract}
RESUMO
$\mathrm{O}$ voluntariado em saúde vem se expandindo em resposta à diversificação das demandas sociais e em contrapartida às contínuas carências do setor. É fundamental, portanto, conhecer a natureza do trabalho voluntário e suas conseqüências para o agente prestador de serviço. Assim, a presente pesquisa teve por objetivos descrever a percepção dos voluntários sobre sua atuação e avaliar o grau de estresse e burnout. Foram realizadas entrevistas estruturadas com 39 voluntários seguidas da aplicação do Inventário de Sintomas de Estresse de Lipp e Guevara e o Inventário de Burnout de Maslach. Verificaram-se baixos escores de estresse e burnout. Contudo, oito voluntários apresentaram sintomas de estresse de natureza psicológica ou somática e quatro revelaram níveis elevados de burnout no que concerne à despersonalização e exaustão emocional. A maioria dos participantes relatou história pessoal vinculada ao adoecimento. Os dados obtidos sugerem a hipótese de que o voluntariado constitui um modo de enfrentamento.
\end{abstract}

Palavras-chave: trabalho voluntário; saúde; auto-percepção; estresse; burnout

\begin{abstract}
Volunteer work in health care: self perception, stress and burnout

Volunteer work in health care is expanding as an answer to the diversification of the social demands and, in counterpart, to the constant lacks in this area. Therefore, it is essential to understand the nature of the volunteer work and its consequences for the provider of this service. The aim of this study was to describe the perception of volunteers about their work and to evaluate the level of stress and burnout in volunteers. Structured interviews were carried out with 39 volunteers, followed by the application of Lipp and Guevara's Stress Symptom Inventory and the Maslach Burnout Inventory. Low scores of stress and burnout were found. However, eight volunteers presented stress symptoms of psychological or somatic nature and four revealed high levels of burnout related to depersonalization and emotional exhaustion. Most of the participants reported personal history factors connected to sickness. The data suggest the hypothesis that the volunteer work constitutes a way of coping.
\end{abstract}

Keywords: volunteer work; health; self-perception; stress; burnout

Definido em lei como atividade não remunerada, prestada por pessoa física a entidade pública de qualquer natureza ou instituição privada de fins não lucrativos, que tenha objetivos cívicos, culturais, educacionais, científicos, recreativos ou de assistência social, o voluntariado vem recebendo cada vez mais atenção dos diferentes setores organizados da sociedade, suscitando um interesse renovado por discussões de caráter acadêmico e científico (Domeneghetti, 2001). Dentre elas, destaca-se a problemática do sofrimento psíquico decorrente do trabalho voluntário e, em especial, aquele realizado na área da saúde e destinado aos cuidados a portadores de câncer e indivíduos soropositivos (Ashforth \& Lee, 1997; Bennett, Ross \& Sunderland, 1996; Jimenez \& Jimenez, 1990; Maslanka, 1996; Nesbitt, Ross, Sunderland \& Shelp, 1996).

Comumente apresentado como fonte de realização pessoal e oportunidade para desempenho de atividades socialmente valorizadas, o voluntariado - compreendido desta maneira - representa um reducionismo com nefastas consequiências no plano individual, institucional e social, na medida em que desconsidera os custos envolvidos e as dificuldades enfrentadas. Deste ponto 
de vista, torna-se essencial ampliar a compreensão sobre a atuação voluntária incluindo seus aspectos favoráveis e desfavoráveis.

É importante lembrar que, quando um indivíduo encontra dificuldades que superam os recursos pessoais ou sociais de adaptação, com freqüência passa a manifestar sinais de estresse e esgotamento (Lipp, 2004). Assim, muitas vezes sem dispor de preparo ou treinamento adequados, o agente voluntário pode se ver exposto a condições inadequadas, comprometendo a si próprio e aqueles a quem deseja ajudar. Do abandono da atividade às interferências prejudiciais na rotina assistencial, várias são as expressões do malestar vivido pelo voluntário.

Há várias décadas, a Psicopatologia do Trabalho focaliza as manifestações de estresse e burnout no campo ocupacional da saúde, paralelamente ao desenvolvimento teórico-metodológico da Psicologia da Saúde, cujo interesse pelo estudo do estresse e do enfrentamento é central em sua delimitação e evolução (Aldwin, 1994; Borges, Argolo, Pereira, Machado \& Silva, 2002; Feliciano, Kovacs \& Sarinho, 2005; Gimenes, 1997; Grunfeld \& cols., 2000; Lipp, 2004; Maslach, Shaufeli \& Leiter, 2001; Murofuse, Abranches \& Napoleão, 2005, Rice, 2000; Stacciarini, 1999; Straub, 2005; Tamayo, 1997; Trucco, Valenzuela \& Trucco, 1999; Tucunduva \& cols., 2006). Contudo, a maior parte dos estudos já realizados estão direcionados para os profissionais da saúde, em particular enfermeiros, e não contemplam o expressivo segmento do voluntariado que atua na área no Brasil.

Provavelmente, em razão da natureza dos cuidados necessários aos portadores de câncer e aos portadores de HIV - envolvendo experiências de risco, discriminação, limitação e até suicídio e morte - o trabalho voluntário nestes serviços é enfatizado pelos pesquisadores. De modo geral, estes estudos visam a caracterização demográfica dos voluntários, suas motivações, modalidades de atuação, necessidades de treinamento, bem como a discussão bioética acerca da solidariedade (Araujo, Maia, Oliveira, 1997; Cassel \& Quellette, 1995; Edgard, Remmer, Rosberger \& Rapkin, 1996; Fusco-Karmann \& Tamburini, 1994; Jimenes \& Jimenes, 1990; Maslanka, 1996; Murrant \& Strathdee, 1995; Nesbitt, Ross, Sunderland \& Shelp, 1996; Paradis \& Usui, 1989; Selli \& Garrafa, 2005; Snyder, Omoto \& Crain, 1999).

Todavia, as informações reunidas até o presente não oferecem uma perspectiva consensual, sendo ne- cessárias mais iniciativas que possam fundamentar ações coerentes e que esclareçam, por exemplo, questões básicas relacionadas aos comportamentos de oferecer e receber ajuda e suas conseqüências no plano pessoal e social. Considerando, então, a inconsistência dos estudos desenvolvidos na área, e particularmente no que se refere à realidade brasileira, a presente investigação teve por objetivos descrever a percepção dos voluntários sobre a sua atuação e avaliar o grau de estresse e burnout.

\section{MÉTODO}

\section{Participantes}

No total, participaram 39 voluntários pertencentes a três instituições de voluntariado voltadas para pacientes oncológicos e a três instituições destinadas ao apoio de portadores do vírus HIV (Moniz \& Araujo, 1999; Souza \& Araujo, 1999). Na área da oncologia, foram estudados 20 voluntários associados ao Movimento de Apoio ao Canceroso (MAC); Serviço Auxiliar Voluntário (SAV) e Associação Brasileira de Apoio às Famílias e Crianças Portadoras de Hemopatias (Abrace). Na área de HIV/aids, foram selecionados 19 voluntários do Grupo de Apoio e Prevenção à Aids (Gapa), Fundação Arco-Íris e Fraternidade Assistencial Lucas Evangelista (Fale).

Todos os participantes da área de oncologia eram do sexo feminino, ao passo que na área de HIV/aids 13 mulheres e seis homens integraram a amostra. Quanto à escolaridade, 19 voluntários tinham nível superior completo e 10 informaram nível superior incompleto. Cinco voluntários tinham o ensino médio incompleto e quatro o ensino médio completo. Uma voluntária possuía pós-graduação em administração. $\mathrm{O}$ número de aposentados $(\mathrm{N}=10)$ predominou na amostra, seguido por estudantes $(\mathrm{N}=7)$, funcionários públicos $(\mathrm{N}=6)$ e donas de casa $(\mathrm{N}=6)$. As profissões de funcionalismo privado e de professor contaram com três representantes cada uma $(\mathrm{N}=6)$. Profissionais liberais (psicólogo e advogado) e outras atividades (pensionista e salgadeira) agruparam dois voluntários cada um $(\mathrm{N}=4)$. Grande parte dos voluntários tinha entre quatro e seis anos de atuação $(\mathrm{N}=17)$, seguidos por aqueles que totalizavam de um a três anos de experiência $(\mathrm{N}=11)$. Cinco voluntários apresentaram menos de um ano de atividade e quatro tinham de sete a nove anos. Duas voluntárias informaram mais de nove anos de trabalho voluntário. 


\section{Procedimentos de coleta e análise dos dados}

Inicialmente, os pesquisadores fizeram um levantamento das principais instituições que prestam serviços voluntários para pacientes oncológicos e soropositivos em hospitais do Distrito Federal e organizações não governamentais. Identificados os contextos representativos para seleção da amostra, iniciou-se a coleta de dados com a realização de entrevistas estruturadas a partir da adaptação do questionário elaborado por Araujo, Maia e Oliveira (1997). Os voluntários foram esclarecidos quanto aos objetivos da pesquisa, assegurados do sigilo e convidados a expressar seu consentimento verbal.

Os relatos foram gravados, transcritos e submetidos a dois procedimentos de análise: a) categorização de acordo com a técnica de análise de conteúdo temática (Bardin, 1977) e b) análise quantitativa do discurso por meio do software Alceste (Analyse Lexicale par Contexte d'un Ensemble de Segments de Texte). Vale explicar que este programa realiza a análise textual e fornece uma classificação estatística de enunciados em função da distribuição de palavras. Após a distribuição em classes, analisam-se qualitativamente os dados com o objetivo de extrair o sentido atribuído ao discurso (Ribeiro, 2000).

Ao final do encontro com os voluntários, foram aplicados: o Inventário de Sintomas de Stress - ISS (Lipp \& Guevara, 1994) e o Inventário de Burnout de Maslach, traduzido, adaptado e validado por Tamayo (1997).

\section{RESULTADOS}

\section{Análise de conteúdo}

Foram elaboradas 10 categorias temáticas: 1) apoio prestado; 2) características e perfil do voluntário; 3) realização pessoal; 4) capacitação e treinamento do voluntário; 5) instituição voluntária e regras formais; 6) percepção da sociedade e do círculo social; 7) relações sociais no hospital; 8) experiência de desgaste e problemas com a atividade; 9) estratégias de enfrentamento; 10) percepção e avaliação da doença e do doente. As instituições foram comparadas a partir da frequiência média de cada categoria. A Abrace apresentou freqüências médias maiores que as outras instituições para cinco das dez categorias.

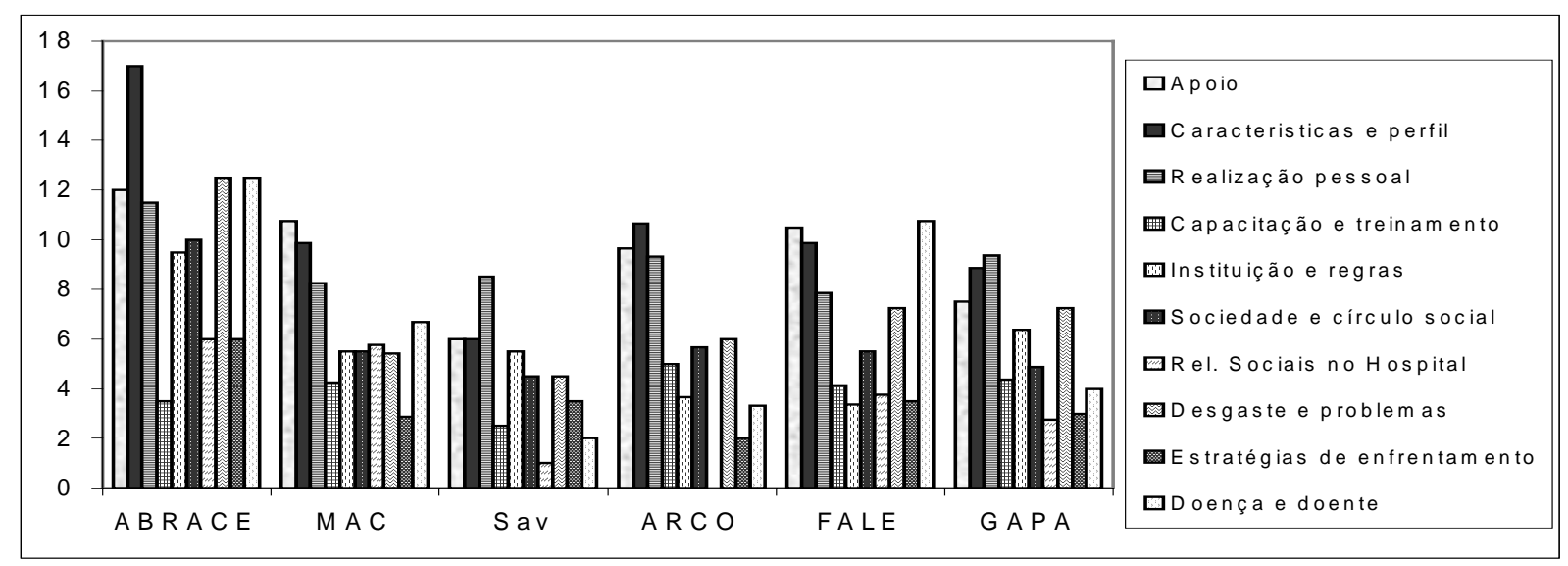

Figura 1. Freqüência das categorias da análise de conteúdo por instituição.

No que diz respeito às instituições que prestam atendimento a portadores do vírus HIV, é interessante salientar que não houve referências dos voluntários da Arco-Íris à interação junto aos profissionais do hospital. Ao se comparar a distribuição das categorias de cada instituição, é possível verificar diferenças que revelam a singularidade de cada uma.
Como se pode observar na figura 2 , há relativa concordância entre os voluntários das duas áreas de atuação quanto à importância das três primeiras categorias. Ao que parece, existe maior diferença entre as instituições do que entre as áreas de atuação voluntária. 


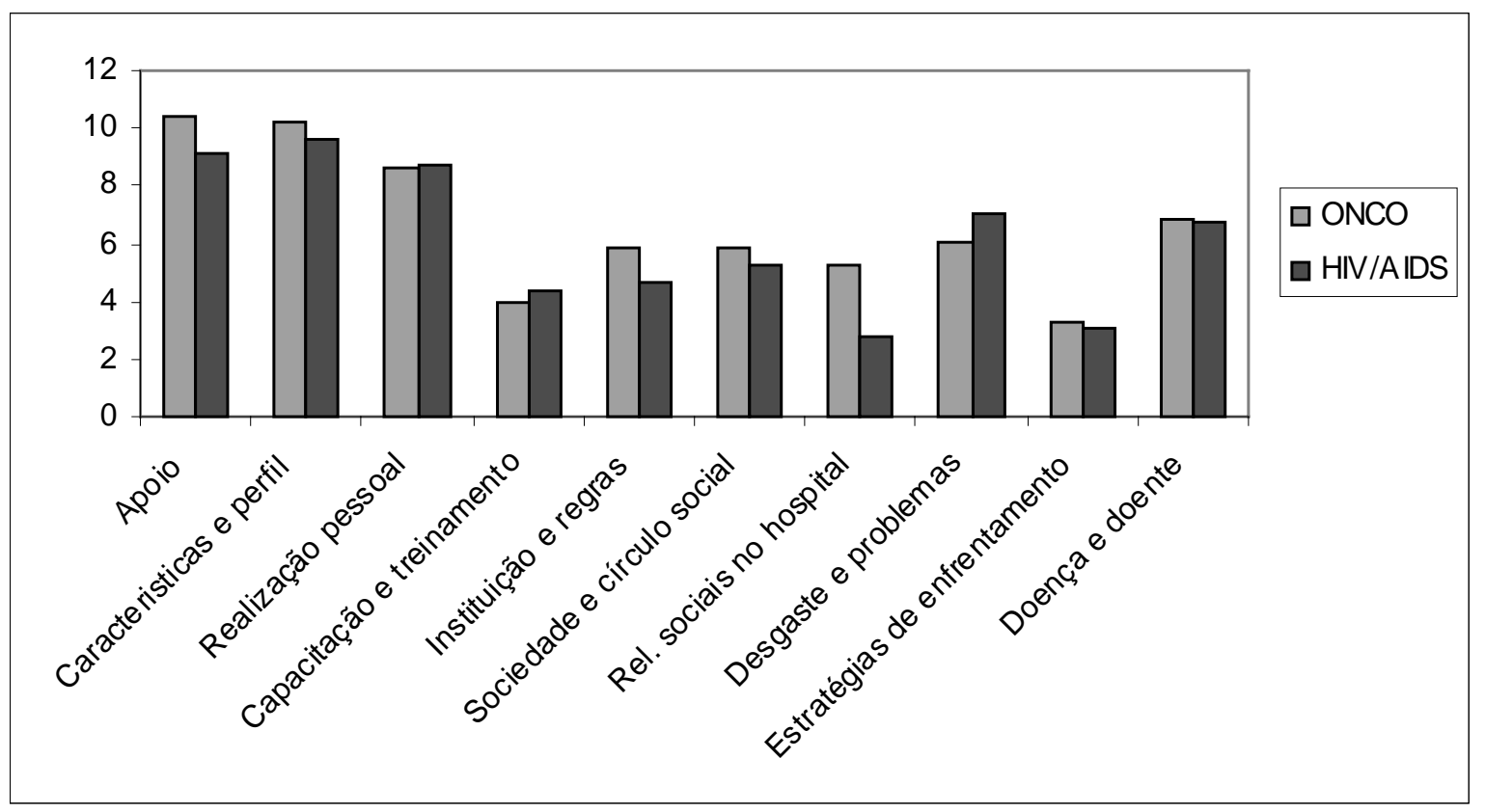

Figura 2. Freqüências das categorias em oncologia e HIV/aids.

\section{Análise pelo Alceste}

O Alceste permitiu identificar seis categorias temáticas nas entrevistas com voluntários. Cada categoria foi definida e exemplificada com frases selecionadas entre aquelas destacadas pelo programa como unidades de contexto. Dois conjuntos distintos de categorias foram identificados: envolvimento anterior e engajamento atual. No primeiro bloco, as categorias experiências e influências anteriores com a doença $\mathrm{e}$ envolvimento emocional com a doença mostraram-se associadas de modo relativamente forte. Este bloco não estabeleceu relação com o bloco engajamento atual, no qual as categorias especificidade do voluntariado e análise social, do sub-bloco avaliação crítica, tiveram associação com outro sub-bloco, chamado organização e provisão, composto pelas categorias instituição voluntária e suporte material. Estas duas últimas mostraram maior associação entre si (Figura 3).

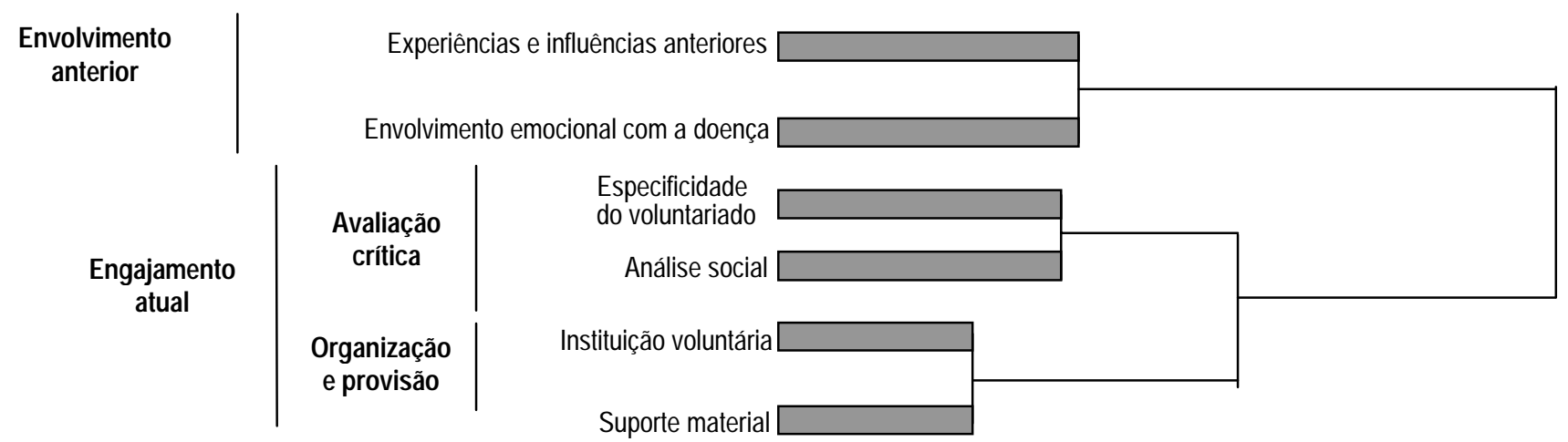

Figura 3. Categorias temáticas dos voluntários segundo a classificação hierárquica descendente fornecida pelo ALCESTE.

Vale explicar que as instituições ABRACE, GAPA e Arco-íris contribuíram conjuntamente para três categorias do bloco engajamento atual, no sub-bloco avaliação crítica e na categoria instituição voluntária. Esta configuração pode demonstrar maior estruturação institucional destas associações, ao mesmo tempo em que se revela maior percepção crítica sobre a sociedade e sobre aquilo que é essencial para tornar-se voluntário. 
A Fale foi destacada como importante para as duas categorias do bloco envolvimento anterior e para análise social, demonstrando maior envolvimento anterior com pacientes soropositivos ou acometidos por outras patologias. O MAC e o SAV, assim como a Fale, também contribuíram para os dois blocos de categorias: envolvimento anterior e engajamento atual, porém em categorias diferentes. O MAC foi destacado para envolvimento emocional com a doença e suporte material. Apenas no MAC houve casos pessoais de câncer entre os voluntários e esta variável também foi importante para as mesmas categorias às quais o MAC foi destacado. Suporte material é uma das principais atividades do MAC (distribuição de cestas básicas e vales-transporte). $\mathrm{O}$ fato destes voluntários, com casos de câncer pessoal, terem destaque nestas categorias pode apontar alguma relação entre o envolvimento emocional com a doença e o suporte material.

Voluntários sem proximidade com doentes foram os mais representativos para o sub-bloco avaliação crítica. No bloco envolvimento anterior constatou-se a participação importante de voluntários como amigos de portadores de HIV/aids e casos pessoais de câncer. Aliás, este foi um fator que teve influência para ingresso no grupo voluntário.

Os estudantes foram mais representativos unicamente na categoria experiências e influências anteriores, o psicólogo em duas categorias do bloco engajamento atual e o professor na categoria análise social. As demais profissões tiveram distribuição equilibrada. Quanto ao tempo de atuação, sobressaíram-se voluntários com menos de um ano de atividade nas duas categorias do sub-bloco organização e provisão.

Vale enfatizar, ainda, que a categoria envolvimento emocional com a doença concentra o maior número de voluntários com sintomas de estresse e de burnout.

\section{Inventários de estresse e de burnout}

A tabela 1 apresenta as médias das respostas de estresse, em suas três fases, para cada instituição. É possível constatar que, de modo geral, as médias situam-se abaixo da pontuação indicada para diagnosticar estresse.

Tabela 1. Média das respostas ao Inventário de Estresse em cada instituição de acordo com as fases de sintomas de estresse

\begin{tabular}{lccc|cccc}
\hline Área & \multicolumn{3}{c|}{ Oncologia } & \multicolumn{4}{c}{ HIV/aids } \\
\hline Fase de estresse & MAC & SAV & ABRACE & GAPA & FALE & Arco-íris & Limites \\
Alerta & 2,18 & 4,5 & 2 & 1,75 & 2 & 2 & 6 \\
Resistência & 2,12 & 1,5 & 2 & 2,25 & 2,5 & 5 & 3 \\
Exaustão & 2,31 & 1 & 1 & 1,5 & 1 & 1 & 8 \\
\hline
\end{tabular}

Tomando-se os voluntários isoladamente, percebeu-se que três membros do MAC apresentaram sintomas predominantemente psicológicos de estresse. Um na fase de alerta e dois na fase de resistência. Um dos voluntários do SAV também expressou sintomas psicológicos de estresse na fase de alerta.

Alguns dos voluntários em HIV/aids também informaram sintomas de estresse significativos. Dois voluntários do Gapa e um da Fale manifestaram sintomas psicológicos de estresse moderado na fase de resistência. Um voluntário da Arco-Íris também co- municou sintomas de estresse alto na fase de resistência predominantemente de natureza física.

A tabela 2 apresenta a média e o desvio padrão das instituições analisadas para cada dimensão de burnout. Verificaram-se médias baixas de exaustão emocional e despersonalização e médias altas do fator diminuição da realização, o que caracteriza um quadro de baixo burnout. É importante lembrar, aqui, que a escala diminuição da realização pessoal do Inventário de Burnout de Maslach é interpretada de forma inversa.

Tabela 2. Média e desvio padrão para exaustão emocional, despersonalização e diminuição da realização por instituição

\begin{tabular}{lcc|cc|cc}
\hline Dimensão & \multicolumn{2}{c|}{ Exaustão emocional } & \multicolumn{2}{c|}{ Despersonalização } & \multicolumn{2}{c}{ Diminuição da realização } \\
\hline Instituição & $\mathrm{M}$ & $\mathrm{Dp}$ & $\mathrm{M}$ & $\mathrm{Dp}$ & $\mathrm{M}$ & Dp \\
MAC & 1,65 & 1,03 & 1,46 & 1,22 & 4,53 & 0,71 \\
SAV & 1,5 & 1,23 & 1,5 & 0,57 & 4,43 & 0,64 \\
ABRACE & 1,45 & 0,94 & 1,5 & 1 & 4,57 & 0,51 \\
GAPA & 2,25 & 1,02 & 2 & 1,41 & 4,12 & 0,63 \\
FALE & 1,92 & 1,14 & 2,75 & 1,58 & 3,82 & 1,02 \\
Arco-íris & 1,39 & 0,87 & 1 & 0 & 3,71 & 0,48 \\
\hline
\end{tabular}


Levantou-se o percentual das respostas de "nunca", "raramente", "algumas vezes", "freqüientemente" e "sempre" para cada uma das dimensões do burnout.
Verificou-se, então, que a grande maioria dos voluntários nunca ou raramente percebe exaustão emocional (ver Tabela 3).

Tabela 3. Avaliação da dimensão de exaustão emocional por instituição

\begin{tabular}{lrrrrr}
\hline $\begin{array}{l}\text { Freqüência/ } \\
\text { Instituição }\end{array}$ & Nunca & Raramente & Algumas vezes & Freqüentemente & Sempre \\
\hline MAC & 63 & 18,8 & 11,6 & 3,3 & 3,3 \\
SAV & 80 & 10 & 0 & 0 & 10 \\
ABRACE & 75 & 15 & 0 & 10 & 0 \\
GAPA & 29,5 & 28,2 & 30,8 & 10,2 & 1,3 \\
FALE & 47,5 & 27,5 & 15 & 5 & 5 \\
Arco & 30 & 60 & 0 & 10 & 0 \\
\hline Total & 53,7 & 22,7 & 14,8 & 5,7 & 3,1 \\
\hline
\end{tabular}

De modo geral, os voluntários nunca ou raramente percebem sintomas de despersonalização (ver Tabela 4). Ainda que a maioria dos voluntários não apresente sintomas de exaustão emocional e de despersonalização, evidenciaram-se algumas avaliações de "freqüientemente" e "sempre" nestas sub-escalas.

Tabela 4. Avaliação da dimensão despersonalização por instituição

\begin{tabular}{lrrrrr}
\hline $\begin{array}{l}\text { Freqüência/ } \\
\text { Instituição }\end{array}$ & Nunca & Raramente & Algumas vezes & Freqüentemente & Sempre \\
\hline MAC & & & & & \\
SAV & 81,3 & 10,7 & 4 & 0 & 4 \\
ABRACE & 60 & 20 & 10 & 10 & 0 \\
GAPA & 70 & 10 & 10 & 0 & 10 \\
FALE & 57,5 & 22,5 & 7,5 & 10 & 2,5 \\
Arco & 45 & 15 & 25 & 10 & 5 \\
\hline Total & 100 & 0 & 0 & 0 & 0 \\
\hline
\end{tabular}

Já na dimensão diminuição da realização, houve maior frequiência percentual de respostas "sempre" e "frequientemente" em todas as instituições, as quais somam mais de $80 \%$ das respostas dadas (ver Tabela 5). Nenhum dos voluntários da área de Oncologia, que apresentou sintomas de estresse, manifestou sintomas de burnout. Três dos quatro voluntários da área de HIV/aids que apresentaram sintomas de estresse também manifestaram sintomas de burnout.

Tabela 5. Avaliação da dimensão diminuição da realização por instituição

\begin{tabular}{lrrrrr}
\hline $\begin{array}{l}\text { Freqüência/ } \\
\text { Instituição }\end{array}$ & Nunca & Raramente & Algumas vezes & Freqüentemente & Sempre \\
\hline MAC & 0 & 1 & 9,7 & 24,3 & 65 \\
SAV & 0 & 0 & 7,1 & 42,9 & 50 \\
ABRACE & 0 & 0 & 0 & 42,9 & 57,1 \\
GAPA & 0 & 0 & 14,2 & 58,9 & 26,9 \\
FALE & 3,6 & 3,6 & 28,5 & 35,7 & 28,6 \\
Arco & 0 & 0 & 28,5 & 71,4 & 0 \\
\hline Total & 0,4 & 0,9 & 13 & 38,3 & 47,4 \\
\hline
\end{tabular}




\section{DISCUSSÃO}

A exemplo da proposta de Zurcher (conforme citado por Rubin \& Thorelli, 1984), a análise dos dados demográficos relativos à formação acadêmica e profissão dos voluntários permite constatações interessantes. Dez voluntários apresentaram relação direta entre a sua formação acadêmica e a área da saúde. Isso pode demonstrar um papel social efêmero em extensão às suas práticas profissionais ou como complemento para a formação. Os demais podem estar buscando desenvolver um papel social compensatório por intermédio das atividades voluntárias. Aposentados e desempregados foram mais freqüentemente destacados para categorias relativas à atuação institucional e capacitação, o que parece demonstrar o papel extensivo da atividade voluntária. Já os estudantes, relacionaram-se predominantemente a categorias voltadas à proximidade atual ou anterior com os pacientes e suas atividades parecem ser de caráter compensatório. A constatação de predominância feminina na amostra investigada pode ser compreendida em razão do papel, culturalmente atribuído à mulher, como cuidadora (Zweigenhaft, Armstrong \& Quintis, 1995).

Predominou uma percepção positiva das características do voluntário como pessoas afetuosas, otimistas, com desprendimento material, estabilidade financeira, disponibilidade de tempo e que fazem um trabalho importante e positivo. A motivação altruísta, destacada no tema interesse em ajudar na categoria características e perfil do voluntário, mostrou-se relacionada a outras motivações que não se associam à recompensa egóica como atender às necessidades externas.

Da mesma maneira que Murrant e Strathdee (1995) não identificaram diferenças entre as motivações dos voluntários em HIV/aids e os demais voluntários do sistema de saúde, a presente investigação não encontrou um tipo de motivação específico para uma ou outra especialidade.

Cinco elementos pareceram significativos para a inserção do voluntário em saúde: a experiência anterior com a doença (pessoal, familiar ou no círculo social), a busca de realização pessoal no atendimento às necessidades pessoais, o interesse em atender às necessidades de um grupo específico (doentes, hospital ou a própria instituição voluntária) e a influência social.

Embora não exista ganho material, há um ganho pessoal no exercício da atividade que se manifesta pelas gratificações associadas a: desenvolvimento, aprendizado e experiência; reconhecimento social (respeito e valorização); mudança de valores pessoais (desprendimento e revisão de preconceitos); senso de utilidade e importância pessoal (ocupação do tempo e contribuição para o grupo); satisfação (prazer pessoal); relação afetiva prazerosa com o atendido; alcance de ideais religiosos, minoração e superação dos próprios problemas e dificuldades, aprimoramento de experiências anteriores, realização de projeto de vida, conquista e ampliação do círculo social.

A meta-percepção dos voluntários é negativa. $\mathrm{Ou}$ seja, consideram que são alvo de críticas por parte da sociedade. Alguns relatam que já foram chamados de "pessoas bobas, anormais, desequilibradas" ou ainda de "aidéticos" e "cancerosos". Mas, quando os voluntários julgam ser percebidos positivamente, esta valorização social também se constitui em um tipo particular de gratificação.

Foram reportados problemas, sentimentos e sensações desagradáveis relacionados com: confronto entre a atividade e a vida pessoal do voluntário, relações difíceis com o doente; conflitos na própria instituição voluntária, dificuldades enfrentadas pelo hospital e dificuldades relacionais com os profissionais de saúde.

De modo similar às constatações feitas por Nesbitt e cols. (1996), os escores de estresse e burnout detectados nesta pesquisa foram baixos. Contudo, sete voluntários (do total de 39) manifestaram sintomas de estresse predominantemente psicológicos. Já a avaliação de burnout encontrou baixos escores para as dimensões exaustão emocional e despersonalização, e altos escores para diminuição da realização pessoal, sendo que quatro voluntários apresentaram sintomas de burnout, três de despersonalização e um de exaustão emocional. Três dos voluntários que indicaram sintomas de burnout também apresentaram sintomas de estresse.

É possível supor que apesar da exposição a diversos estressores, os voluntários contam com estratégias de enfrentamento, que eliminam ou aliviam os sintomas de estresse e burnout, de modo tal que persistem na atividade. Levando-se em consideração que o tempo de atuação da maioria dos participantes varia de um a seis anos, pode-se cogitar que têm sido efetivas as estratégias adotadas, a saber: preparo psicológico $e$ técnico, crença religiosa, expressão aberta das insatisfações, uso de gratificações e recompensas como compensação ao estresse sofrido, cumprimento das orientações da direção do grupo, crença de que os problemas fazem parte do trabalho, senso de utilidade e crença de que a morte constitui um alivio para os pacientes. 
Quando se trata de capacitação, as categorias de instituição voluntária e regras formais e as relações sociais no hospital, sobretudo com profissionais, e a capacitação e treinamento do voluntário aproximamse. Em outras palavras, de um lado, as associações voluntárias têm regras, exigências e proibições que devem ser respeitadas pelo agente voluntário. De outro lado, as relações que este estabelece com outros profissionais, embora restritas, apresentam-se como fonte de parcerias ou críticas. É importante esclarecer que, freqüentemente, os voluntários encontram-se vinculados ao profissional do serviço social que é encarregado da organização e controle do trabalho voluntário.

A categoria capacitação do voluntário surgiu de modo evidente nas análises de conteúdo das entrevistas. Os voluntários também reportaram necessidade de mais informações sobre prevenção e drogadição, além de mais prática e acompanhamento. Tal constatação pode ser melhor compreendida à luz das reflexões feitas por Fusco-Karman, Gangeri, Tamburini e Tinini (1996), de que muitos voluntários envolvem-se com o suporte emocional do paciente, o que pode levar ao reconhecimento da necessidade de treinamento para atender tal função. Então, apesar de cinco dentre as seis instituições estudadas fornecerem treinamento para seus voluntários, persistem outras necessidades decorrentes da interação com o paciente. É crucial pesquisar em maior profundidade esta questão, acompanhando e avaliando os programas de capacitação e formação fornecidos no âmbito das associações.

\section{CONSIDERAÇÕES FINAIS}

Sugere-se que futuras pesquisas ampliem o foco adotado no presente trabalho e investiguem a percepção dos profissionais de saúde, dos "assistidos" e do público em geral sobre a atuação voluntária. Parece essencial, também, estudar de modo articulado motivação e gratificação, pois a primeira é importante para o ingresso na atividade, enquanto a segunda influencia na sua permanência.

Considera-se fundamental estabelecer meios de avaliação criteriosos que envolvam não apenas a utilidade e a qualidade destes serviços, mas que incluam também parâmetros relativos à saúde física e mental do agente voluntário a partir de uma perspectiva preventiva. Embora a grande maioria dos participantes deste estudo não tenha manifestado graves sintomas de estresse e burnout, foram detectadas dificuldades que merecem atenção. Uma vez mais, é necessário insistir quanto à importância do suporte e orientação adequadamente planejados para o acompanhamento do trabalho voluntário.

Reportado como gratificante, embora existam insatisfações, é possível hipotetizar que o voluntariado reveste-se como um valor e constitui-se como uma estratégia de enfrentamento (Aldwin, 1994; Gimenes, 1997). Tal condição diminui o impacto negativo do sofrimento psíquico do agente contribuindo para mantê-lo em atividade. De acordo com Midlarsky (1991), a ajuda prestada em condição de estresse gera sensações de bem-estar e competência, levando à reavaliação do estresse que passa a ser percebido como desafio, ao invés de ameaça ou perda. Em síntese, o estudo do comportamento de ajuda como uma modalidade de enfrentamento exige aprofundamento, especialmente na esfera da assistência à saúde e, nesse caso, o voluntariado apresenta-se como temática de relevância.

\section{REFERÊNCIAS}

Aldwin, C. M. (1994). Stress, coping and development: an integrative perspective. New York - London: Guilford Press.

Araujo, T. C. C. F., Maia, L. M. E. \& Oliveira, D. S. (1997). Voluntariado em oncologia: Estudo exploratório [Resumo]. Em VII Encontro Nacional dos Psicólogos da Área Hospitalar (Org.), Resumos de comunicações científicas (p. 44). Brasília: Universidade de Brasília.

Ashforth, B. E. \& Lee, T. R. (1997). Burnout as a process: commentary on Cordes, Dougherty and Blum. Journal of Organizational Behavior, 18, 703-708.

Bardin, L. (1977). Análise de conteúdo. Portugal: Edições 70.

Bennett, L., Ross, M. W. \& Sunderland, R. (1996). The relationship between recognition rewards and burnout in AIDS caring. AIDS Care, 8, 145-153.

Cassel, J. B. \& Quellette, S. (1995). A tipology of AIDS volunteers. Aids Education and Prevention, 4, suplement, 80-90.

Domeneghetti, A. M. (2001). Voluntariado: gestão do trabalho voluntário em organizações sem fins lucrativos. São Paulo: Esfera.

Edgar, L., Remmer, J., Rosberger, Z. \& Rapkin, B. (1996). An oncology volunteer support organization: the benefits and fit within the health care system. Psycho-Oncology, 5, 331-341.

Feliciano, K. V. O., Kovacs, M. H. \& Sarinho, S. W. (2005). Sentimentos de profissionais dos serviços de pronto-socorro pediátrico: reflexões sobre o burnout. Revista Brasileira de Saúde Materno Infantil, 5, 319-328.

Fusco-Karmann, C. \& Tamburini, M. (1994). Volunteers in hos-

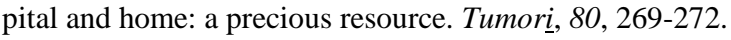

Gimenes, M. G. (1997). A teoria do enfrentamento e suas implicações para sucessos e insucessos em psiconcologia. Em M. G. Gimenes \& M. H. Fávero (Org.), A mulher e o câncer (pp. 111147). Campinas: Editorial Psy. 
Grunfeld, E., Whelan, T. J., Zitzelsberger, L., Willian, A. R., Montesanto, B. \& Evans, W. K. (2000). Cancer care workers in Ontario: prevalence of burnout, job stress and job satisfaction. Canadian Medical Association Journal, 163, 166-169.

Jimenez, M. A. \& Jimenez, D. R. (1990). Training volunteers caregivers of persons with AIDS. Social Work in Health Care, $14,73-85$.

Lipp, M. E. N. (Org.) (2004). Stress no Brasil: pesquisas avançadas. Campinas: Papirus.

Lipp, M. E. N. \& Guevara, A. H. (1994). Validação empírica do Inventário de Sintomas de Stress. Estudos de Psicologia, 11, 43-49.

Maslach, C., Shaufeli, W. B. \& Leiter, M. P. (2001). Job burnout. Anual Reviews of Psychology, 52, 397-422.

Maslanka, H. (1996). Burnout, social support and AIDS volunteers. AIDS Care, 8, 195-206.

Midlarsky, E. (1991). Helping as coping. Em M. S. Clark (Org.), Prossocial behavior (pp. 238-263). California: Sage.

Moniz, A. L. F. \& Araujo, T. C. C. F. (1999). Atuação voluntária em serviços de oncologia. [Resumo]. Em $5^{\circ}$ Congresso de Iniciação científica da Universidade de Brasília (p. 449). Brasília: Universidade de Brasília.

Murofuse, N. T., Abranches, S. S. \& Napoleao, A. A. (2005). Reflexões sobre estresse e burnout e a relação com a enfermagem. Revista Latino-Americana de Enfermagem, 13, 255-261.

Murrant, G. \& Strathdee, S. A. (1995). Motivations for service volunteer involvement at casey house AIDS hospice. The Hospice Journal, 10, 27-38.

Nesbitt, W. H., Ross, M. W., Sunderland, R. H. \& Shelp, E. (1996). Prediction of grief and HIV/AIDS-related burnout in volunteers. AIDS Care, 8, 137-143.

Paradis, L. F. \& Usui, W. N. (1989). Hospice staff and volunteers: issues for management. Journal of Oncology, 7,121-140.

Ribeiro, A. S. M. (2000). Alceste: análise quantitativa de dados textuais. Manual não publicado. Laboratório de Psicologia Escolar, Universidade de Brasília, Brasília.

Rice, V. H. (2000). Theories of stress and relationship to health. Em V. H. Rice, (Org.), Handbook of stress, coping, and health: implications for nursing research, theory and practice (p. 2745). Canada: Thousand Oaks, Sage.

Rubin, A. \& Thorelli, I. M. (1984). Egoistic motives and longevity of participation by service volunteers. Journal of Applied Behavioral Science, 20, 223-235.

Selli, L. \& Garrafa, V. (2005). Bioética, solidariedade crítica e voluntariado orgânico. Revista de Saúde Pública, 39, 473-478.

Snyder, M., Omoto, A. M. \& Crain, A. L. (1999). Punished for their good deeds: stigmatization of AIDS volunteers. American Behavioral Scientist, 42, 1.175-1.192.

Souza, M. R. \& Araujo, T. C. C. F. (1999). Atuação voluntária em unidades de cuidados a portadores do vírus HIV/aids [Resumo]. Em $5^{\circ}$ Congresso de Iniciação científica da Universidade de Brasília (p. 451). Brasília: Universidade de Brasília.

Stacciarini, J. M. (1999). Estresse ocupacional, estilos de pensamento e coping na satisfação, mal-estar físico e psicológico dos enfermeiros. Tese de Doutorado não publicada, Universidade de Brasília, Brasília.

Straub, R. (2005). Psicologia da saúde. Porto Alegre: Artmed.

Tamayo, M. R. (1997). Relação entre a síndrome do burnout e os valores organizacionais no pessoal de enfermagem de dois hospitais públicos. Dissertação de Mestrado não publicada, Universidade de Brasília, Brasília.

Trucco, M. B., Valenzuela, P. A. \& Trucco, D. H. (1999). Estrés ocupacional en personal de salud. Revista Médica do Chile, $127,1.453-1.461$.

Tucunduva, L. T. C. M., Garcia, A. P., Prudente, F. V. B., Centofanti, G., Souza, C. M., Monteiro, T. A., Nince, F. A. H., Samano, E. S. T., Gonçalves, M. S. \& Del Giglio, A. (2006). A síndrome da estafa profissional em médicos cancerologistas brasileiros. Revista da Associação Médica Brasileira, 52, 108112.

Zweigenhaft, R. L., Armstrong, A. \& Quintis, F. (1995). The motivations and effectiveness of hospital volunteers. Journal of Social Psychology, 136, 1, 25-34.

Recebido: $25 / 01 / 2006$ Revisado: 11/09/2006 Aceito: $11 / 10 / 2006$

\section{Agradecimentos}

Agradecemos à Prof ${ }^{a}$. Angela Maria de Oliveira Almeida e ao Dr. Sandro Aldry Monteiro pelo valioso apoio na utilização do programa Alceste, assim como às auxiliares de pesquisa Mariana Lobo e Adriana Melquíades e à bolsista de iniciação científica Marizaura Souza pela participação na coleta e análise dos dados.

\section{Sobre os autores:}

André Luís Ferreira Moniz: Autor da pesquisa, sob a orientação da segunda autora, com Bolsa da Coordenação de Aperfeiçoamento de Pessoal de Nível Superior - Capes.

Tereza Cristina Cavalcanti Ferreira de Araujo: Pesquisadora do Conselho Nacional de Desenvolvimento Científico e Tecnológico CNPq.

Endereço para correspondência: Universidade de Brasília, Instituto de Psicologia, Laboratório de Saúde e Desenvolvimento Humano, Campus Darcy Ribeiro, Asa Norte - 70910-900 Brasília/ DF. Endereço eletrônico: amoniz@ superig.com.br ou araujotc@unb.br. 